Z Epileptol 2014 - 27:161-161

DOI 10.1007/s10309-014-0368-0

Online publiziert: 6. April 2014

(c) Springer-Verlag Berlin Heidelberg 2014

G. Kurlemann

Klinik für Kinder-Jugendmedizin, Allgem. Kinderheilkunde, Bereich Neuropädiatrie, Münster

\title{
Stoffwechsel und Epilepsie
}

Schwerpunkt ein: Innerhalb der verschiedenen Stoffwechselerkrankungen werden der Glucosetransporterdefekt und die BVitamine mit ihrer Bedeutung für die Epilepsie und das gesamte Fach der Neuropädiatrie sowie Neurologie in jeweils gesonderten Beiträgen dargestellt. Das Spektrum der „übrigen“ Stoffwechselstörungen, bei denen nicht selten der epileptische Anfall oder das klinische Bild einer epileptischen Enzephalopathie als Erstsymptom im Vordergrund steht, wird nach dem Alter der Erstmanifestation in 2 Gruppen unterteilt: metabolische Epilepsien innerhalb der Neonatalperiode (Geburt bis zum 28. Lebenstag) und metabolische Epilepsien vom Säuglingsüber das Kleinkind- bis zum Jugendalter.

Die Stoffwechselerkrankungen des Erwachsenenalters, seltener als im Kindesalter, sollen nicht unerwähnt bleiben und finden genauso ihre Beachtung, um das gesamte Feld metabolischer Epilepsien darzustellen.

Klinische Leitsymptome wie fazialbetonte hochfrequente Myoklonien bei Pyridoxalabhängigkeit oder bestimmte Körpergerüche - z. B. nach Schweißfüßen bei der Isovalerianacidurie oder okulogyre Krisen beim L-Aminosäuren-Decarboxylase-Mangel sind klinische Leitsymptome, die an eine metabolische Genese denken lassen, wie das EEG mit richtungweisenden Befunden für die Diagnose angeborener Stoffwechselstörungen mit epileptischen Anfällen gerade im Kindesalter. Diese wenigen, aber wichtigen richtungweisenden EEG-Muster runden dieses Heft der Zeitschrift für Epileptologie zum Thema Stoffwechsel und epileptische Anfälle $a b$.
Wir erhoffen uns, mit diesem Sonderheft einen raschen Zugang zu den Erkrankungen aus dem Formenkreis der angeborenen Stoffwechselstörungen mit epileptischen Anfällen überhaupt und deren Differenzialdiagnose zu ermöglichen.

Gerhard Kurlemann

\section{Korrespondenzadresse}

Prof. Dr. G. Kurlemann

Klinik für Kinder-Jugendmedizin,

Allgem. Kinderheilkunde,

Bereich Neuropädiatrie

Albert-Schweitzer-Campus 1,

Gebäude 1A, 48149 Münster

Gerhard.Kurlemann@ukmuenster.de

\section{Einhaltung ethischer Richtlinien}

Interessenkonflikt. G. Kurlemann gibt an, dass kein Interessenkonflikt besteht. 